In univariate Cox regression analyses, PASP, NYHA and LVEF, at 1-month following TAVI, all predicted 1-year mortality $(\mathrm{p}<0.05$ ). In multivariate analysis, only NYHA at 1month following TAVI was independently related to 1-year mortality (hazard ratio $1.80,95 \%$ confidence interval 1.21 to 2.69, $\mathrm{p}=0.004)$.

Conclusion These data provide evidence that the reduction in PASP observed following TAVI is closely correlated with an improvement in NYHA functional class along with mortality at 1-year. This study will help enable cardiovascular clinicians to identify those patients likely to have a favourable symptomatic response to TAVI based on the echocardiographic PASP estimate post-procedure.

\section{INADEQUACY OF EXISTING CLINICAL PREDICTION MODELS FOR PREDICTING MORTALITY POST TRANSCATHETER AORTIC VALVE IMPLANTATION}

${ }^{1}$ Glen Martin*, ${ }^{1}$ Matthew Sperrin, ${ }^{2}$ Peter Ludman, ${ }^{3}$ Mark de Belder, ${ }^{4}$ Chris Gale, ${ }^{5}$ William Toff, ${ }^{1}$ lain Buchan, ${ }^{1}$ Mamas Mamas. ${ }^{1}$ University of Manchester, ${ }^{2}$ Queen Elizabeth Hospital; ${ }^{3}$ James Cook University Hospital; ${ }^{4}$ Leeds Institute of Cardiovascular and Metabolic Medicine; ${ }^{5}$ University of Leicester; * Presenting Author

\subsection{6/heartjnl-2016-309890.47}

Introduction Clinical prediction models (CPMs) form the cornerstone of risk stratification for patients undergoing invasive procedures, helping to guide both treatment allocation and the consent process by enabling discussion of the magnitude of risks associated with the proposed treatment strategy. Several CPMs are used to predict mortality after Transcatheter Aortic Valve Implantation (TAVI). However, their performance in national cohorts distinct from those where they have been derived is unknown. We examined the performance of the Logistic EuroSCORE (LES), EuroSCORE II, Society of Thoracic Surgeons (STS), German Aortic Valve, FRANCE-2 TAVI and OBSERVANT-TAVI risk models in the British Cardiovascular Intervention Society UK TAVI registry, to examine if any can be used for practical use in external national cohorts of TAVI patients.

Methods The risk of 30-day mortality given by each CPM was retrospectively calculated, based on the published regression coefficients, for all 3980 patients in the UK TAVI registry from 2007 to 2012. Life status information was provided by the Office for National Statistics for each patient. Performance of each CPM was investigated through calibration and discrimination. Calibration is the agreement between the expected and observed event rates and was analysed by a calibration plot. Discrimination is how well the CPM can distinguish between those who will experience an event and those who will not, which was analysed by the area under the ROC curve (AUC). Furthermore, the level of agreement in risk classification between each of the considered CPMs was analysed at an individual patient level.

Results The observed 30-day mortality rate was $6.23 \%$. All of the CPMs were miscalibrated and grossly over-predicted risk of 30-day mortality; the LES over predicted by a factor of three, while the STS underestimated risk. Discrimination was also poor, with AUC values of 0.58, 0.57, 0.62, 0.60, 0.62 and 0.57 for the LES, EuroSCORE II, STS, the German Aortic Valve, FRANCE-2 TAVI and OBSERVANT models, respectively. Additionally, risk classification agreement was low in both the surgical models and the TAVI specific models, an example is given in Figure 1.

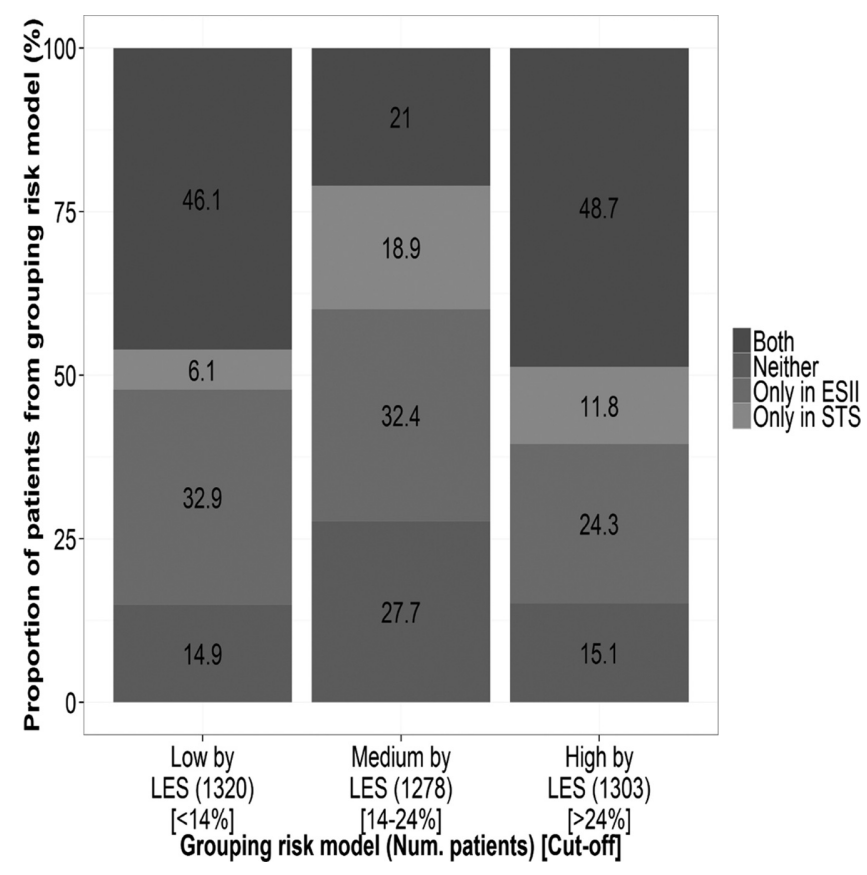

Abstract 47 Figure 1 Bar plot giving the proportion of patients that agree in risk allocation based on the LES. The cut-off values were set to give approximately equal numbers of patients in each risk strata

Conclusions The performance of CPMs currently used in TAVI risk prediction was low when applied in a cohort of TAVI patient independent to those they were developed on. The TAVI specific FRANCE-2 CPM performed better than cardiac surgery based CPMs. Thus, development of TAVI CPMs are recommended either by updating existing CPMs or developing new risk scores in populations of interest.

\section{BCS Affiliates - allied professionals (BACPR/ BANCC)}

\section{DETECTION AND MANAGEMENT OF ATRIAL FIBRILLATION IN THE PACING CLINIC}

${ }^{1}$ Mark Gilmore, ${ }^{2}$ James Barry, ${ }^{3}$ Paul Rees*, ${ }^{4}$ Wendy Churchouse. ${ }^{1}$ Morriston Hospital - EP Fellow - Cardiology; ${ }^{2}$ Morriston Hospital - Consultant Cardiologist; ${ }^{3}$ Morriston Pacemakerl Defibrillator Clinic; ${ }^{4}$ Morriston Hospital - Arrhythmia Nurse Specialist; *Presenting Author

\subsection{6/heartjnl-2016-309890.48}

Purpose Atrial fibrillation (AF) is a common arrhythmia and is a significant risk factor for stroke. Oral anticoagulants are effective treatments which substantially lower the risk of stroke in patients with AF. Sadly many patients are not prescribed anticoagulants.

Modern pacemakers are capable of acting as cardiac monitors and store episodes of AF or mode switch events (MS) which may indicate the presence of AF. Utilisation of this pacemaker technology by cardiac physiologists could improve early detection and treatment of $\mathrm{AF}$ improving patient outcomes and reduce stroke risk.

The audit aimed to investigate the frequency of AF in our pacemaker population and determine whether appropriate 
action had been taken to identify the episodes and notify the general practitioner (GP).

Method A retrospective analysis of pacing clinic notes was made for 100 consecutive patients attending pacemaker interrogation.

12 NEW cases of AF were diagnosed from the utilisation of pacemaker technology.

$1(8 \%)$ of the 12 was already anticoagulated and documented.

$2(17 \%)$ patients - no clear documentation of action taken e.g. GP informed.

$9(75 \%)$ patients - clear documentation that GP had been informed and action had been taken.

17 patients had episodes of MS greater than $30 \mathrm{~s}$.

$10(59 \%)$ had intracardiac electrogram monitoring (EGM) activated and returned to clinic in two months time.

$1(6 \%)$ patient had an older device with no EGM monitoring available and was fitted with a holter monitor.

$6(35 \%)$ patients had no clear plan documented.

A repeat retrospective analysis of 100 patients was made 6 months later and the results showed:

19 NEW cases of AF were diagnosed.

$3(16 \%)$ of the 19 was already anticoagulated and documented.

$16(84 \%)$ patients - clear documentation that GP had been informed and action had been taken.

All patients were appropriately managed.

16 patients had episodes of MS greater than $30 \mathrm{~s}$.

$13(81 \%)$ had EGM activated and returned to clinic in two months time.

$1(6 \%)$ patient had an older device with no EGM monitoring available and was already anticoagulated.

$2(13 \%)$ patients were nearing battery replacement and EGM storage was not activated to conserve battery life.

Conclusion AF is highly prevalent in our pacemaker population. Utility of pacemaker technology by cardiac physiologists improved early detection and treatment of $\mathrm{AF}$ in $83 \%$ patients. This improved patient outcomes and reduced stroke risk significantly.

Future improvements included a clear, auditable pathway for physiologists when AF is suspected/identified to ensure this is consistently communicated to patients and their doctors. This is reflected from the repeat retrospective study which has shown raising awareness can have an impact.

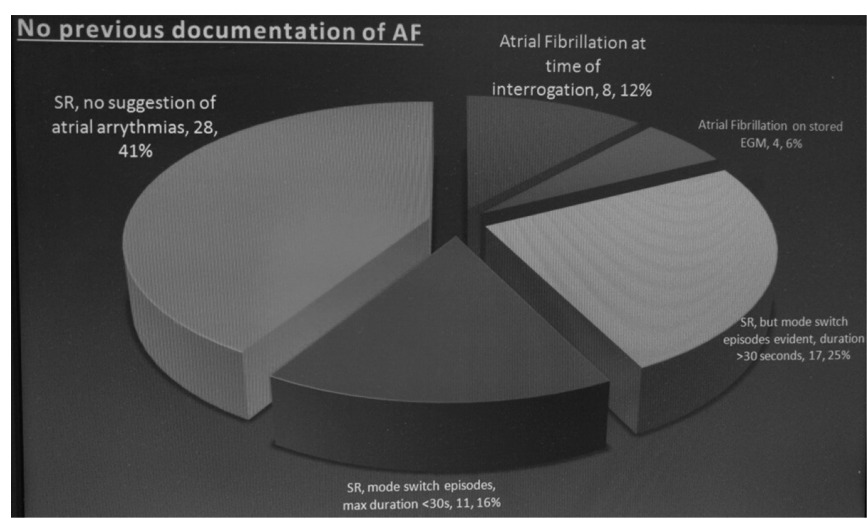

Abstract 48 Figure 132 of the 100 patients were diagnosed with AF previously

\section{Cardiac Rhythm Management}

\section{RAPID ACCESS AF CLINIC - A NEW SERVICE TOWARDS BETTER OUTCOME}

${ }^{1}$ Baskar Sekar*, ${ }^{2}$ Jonathon Whyler, ${ }^{2}$ Emma Williams, ${ }^{2}$ Abdul Azzu, ${ }^{2}$ Mark Payne, ${ }^{2}$ Azad Hanna. 'New Cross Hospital; ${ }^{2}$ Ysbyty Gwynedd Hospital ${ }^{*}$ Presenting Author

\subsection{6/heartjnl-2016-309890.49}

Introduction Atrial fibrillation (AF) is one of the top ten reasons for hospital admissions. Failure to recognise this arrhythmia and risk stratify patients early on diagnosis can have detrimental consequences including high risk of thromboembolic events, stroke, heart failure and death. An audit conducted in our hospital (2011-2012) revealed that patients with AF stay an average 5.6 days in hospital. It has been estimated that billions of pounds are spent each year from health and social care budgets due to AF and AF related strokes. ${ }^{1}$ To address this, a new service "Rapid access AF clinic (RAAFC)" was developed in our hospital in June 2012. This retrospective study explores the role of RAAFC on clinical outcomes since its introduction.

Methods 210 patients were seen in our clinic between 01/06/ 2012 and 30/10/2015. 56 patients were excluded from the analysis - - 41 due to lack of access to records, 1 found to be in $\mathrm{CHB}$ and 14 in sinus rhythm. 154 patients were

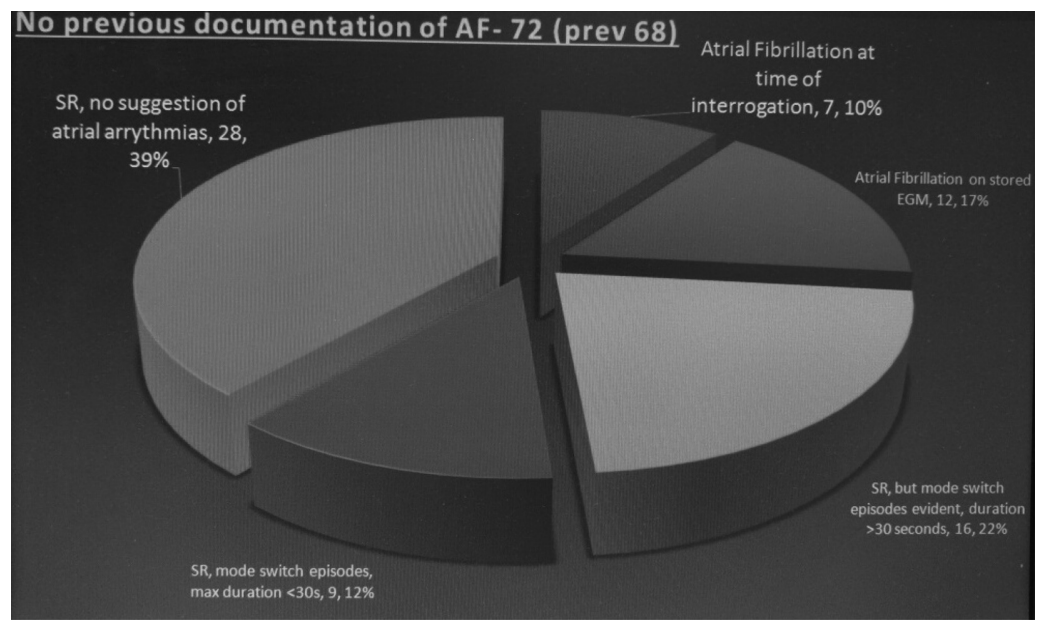

Abstract 48 Figure 228 of the 100 patients were diagnosed with AF previously 